\title{
The cold genesis-a new scenario of particles forming
}

\begin{abstract}
There are presented briefly the main theoretic models of the theory developed by author in the book: "The cold Genesis of Matter and Fields" (Ed. Science PG, 2015), which argues the cold genesis of elementary particles in a very strong magnetic field, comparable to those of a magnetar or a gravistar. The elementary particles are explained by a quasi-crystalline model of quark, resulted as Bose-Einstein condensate of gammons considered as $\mathrm{N}$ pairs of quasi-electrons with diminished $m_{e}$-mass, $e^{*}$-charge and $\mu_{e}^{*}\left(\Gamma_{\mu}^{*}\right)$-magnetic moment. The nuclear force is explained as attraction of the nucleon's impenetrable volume in the field of $2 \mathrm{~N}$ - superposed magnetic moment vortices $\Gamma_{\mu}^{*}(r)$ of another nucleon. The theory predicted the existence of a preon $z^{0} \approx 34 m_{e}$ with quasi-crystalline kernel, experimentally evidenced in 2015 but considered as X-boson of a fifth force, and the field-like nature of the dark energy.
\end{abstract}

Volume | Issue 5 - 2017

\section{Marius Arghirescu}

Patents Department, State Office for Inventions and Trademarks, Romania

Correspondence: Marius Arghirescu, Patents Department, State Office for Inventions and Trademarks, Bucharest, Romania, Email arghirescu.marius@osim.ro

Received: September 30, 2017 | Published: November 17, 2017

Keywords: cold genesis, bose-einstein condensate, quasi-crystal quark, dark energy, quantum vortex

\section{Commentary}

According to a relative recent paper, ${ }^{1}$ a new neutral boson of mass $\sim 17 \mathrm{MeV} / \mathrm{c}^{2},(\sim 34 \mathrm{~m})$, was experimentally evidenced by a hungarian research team by generating an excited $\mathrm{Be} 8 *$ nuclear state, obtained by proton interaction with a target of Li7; but this boson was supposed to be an X-boson of a fifth basic force, of leptons to quarks binding, by a team of american physicists. ${ }^{2}$ The existence of this boson was predicted by a cold genesis theory of matter, developed by author in the book: "The cold Genesis of Matter and Fields", 3,4 as being a basic $z^{0}$ preon of cold formed quarks, resulted as quasicrystallin cluster of paired quasi-electrons, i.e-with quasi-crystallin preonic structure of its kernel, formed by the super-dense centroids of the component quasi-electrons and contained by its current quark mass.

The main theoretical models of the mentioned cold genesis theory, (CGT), are the next:

According to this theory, based on the galileian relativity, the magnetic field is generated by an etherono-quantonic vortex $\Gamma_{M}=\Gamma_{\mathbf{A}}+\Gamma_{\mu}$ of s-etherons (sinergons-with mass $m_{s} \approx 10^{-60} \mathrm{~kg}$ )- giving the magnetic potential $\mathbf{A}$ by an impulse density: $p_{s}(r)=\left(\rho_{s} \times c\right)_{r}$ and of quantons (h-quanta, with mass: $\left.m_{h}=h / c^{2} \approx 7.37 \times 10^{-51} \mathrm{~kg}\right)$ - giving the magnetic moment and the magnetic induction $\mathbf{B}$ by an impulse density: $p_{c}(r)=\left(\rho_{c} v_{c}\right)_{r}$, generated by a magnetic moment of an atomic particle but also by a magnet or an electromagnet $B_{j}=k_{1}\left(\rho_{c} v_{c}\right)_{r}$.

a. The laws of electromagnetism and of gravitation results in an unitary form which for the gravitation force is

$F_{i}^{g}=m_{p} a_{G i}=q_{G} \cdot E_{G}\left(r, Q_{G}\right)=-k_{h} \cdot m_{p}\left(\rho_{c g} \mathrm{v}^{2} \mathrm{cg} g \rho_{c g}\left\langle\mathrm{v}_{\mathrm{cg}} \cdot \mathrm{v}_{0}\right\rangle\right) \cdot n_{i} ; \mathrm{k}_{\mathrm{h}}=S_{h} / 2 m_{h}$ with: $\mathrm{q}_{\mathrm{G}}=S_{g}^{0} / k_{1} ; \mathrm{k}_{1}=4 \pi \mathrm{a}^{2} / \mathrm{e}=\left(\mathrm{m}_{\mathrm{e}} / \mathrm{e}\right) \cdot \mathrm{k}_{\mathrm{h}} ; \mathrm{E}_{\mathrm{G}}= \pm k_{\mathrm{l}} \rho_{c g} c^{2} ; \mathrm{S}_{\mathrm{g}}^{0}=k_{h} m_{p} ; \mathrm{v}_{\mathrm{o}}\left(q_{G}\right) \perp r$

b. By a static charge model with spheric spatial distribution of field quanta (gravitons: $m_{g} \approx 10^{-69} \mathrm{~kg}$, respective- vectorial photons (vectons): $m_{v} \approx 2.3 \times 10^{-40} \mathrm{~kg}$ ).

c. The theory deduces also a variation of the Compton radius of the fermion's magnetic moment, (i.e- also the magnetic moment variation), inverse proportional with the density in which is placed the particle's super-dense kernel (the particle's centroid).

d. The theory sustains also the possibility of a cold genesis of particles: neutrins, photons, electrons, mesons, baryons, in a very strong magnetic field of a magnetar or a gravistar, with $B_{T} \rightarrow 10^{12} \mathrm{~T}$, in a 'vortexes cascade' particles forming process, by masses given according to the sum rule and equal or very close to a value given as integer number of basic preons $z^{0}$, the elementary particles resulting theoretically in a chiral soliton model as Bose-Einstein condensate of photons with common superdense centroid- in the electron's case and of "gammons" $\gamma_{c}=\left(e^{+}-e^{-}\right)$-considered as pairs of $N^{p}$ degenerate electrons, i.e- of quasi-electrons (with degenerate e-charge, $m$-mass and $\mu_{e}$ - magnetic moment)- in the case of mesons and of baryons, with the inertial mass $m^{*}$, formed by a superdense centroid and a quantum volume of vexons (vectorial photons composed by vortexed vectons- considered as electric field quanta), the particle's magnetic moment radius being given by its Compton radius, $r_{\lambda}=\lambda / 2 p=\hbar / m_{p}^{c}$, according to the known relation $\mu=\left(\right.$ e.c. $\left.r_{\lambda}\right) / 2$.

e. The virtual radius: $r_{m}{ }^{n}$ of the proton's magnetic moment, $\mu$ results - in the theory, by a degenerate Compton radius of $^{p}$ the attached positron, which decreases when the protonic positron is included in the $N^{p}$ cluster volume, from the value: $r^{e}=3.86 \times 10^{-13} \mathrm{~m}$, to the value: $r_{i}=r^{p}=0,59 \mathrm{fm}$, as $a^{m}$ consequence of the increasing of the impenetrable quantum volume mean density in which is included the protonic positron centrol: $m_{0}$, from the value: $\bar{\rho}_{e}$ to the value: $\bar{\rho}_{n} \cong f_{d} \times N^{p} \times \bar{\rho}_{e}$, in which: $k_{P}=\bar{\rho}_{n} / \bar{\rho}_{e}$-the gyromagnetic ratio; $\bar{\rho}_{n} ; \bar{\rho}_{e}$-the mean 
density of electron and nucleon; $f_{d}$-the degeneration coefficient of the quasielectron mass, $m *$. The theory gives an electron radius: $\mathrm{a}=1.41 \mathrm{fm}$ and a quanta density variation inside the electron's quantum volume: $\rho_{e}=\rho_{e}{ }^{0} . e^{r / n}$; with: $\rho_{e}^{0}=22.24 \mathrm{~kg} / \mathrm{m}^{3}$ and: $\eta=0.965 \mathrm{fm}$ for the electron mass quanta density variation.

f. The superposition of the $\left(N^{p}+1\right)$ quantonic vortices: $\Gamma^{*}$ of the protonic quasielectrons generates inside a volume with the radius: $r_{\mu}{ }^{a}=2.35 \mathrm{fm}$, a total dynamic pressure: $P_{n}=(1 / 2) \rho_{n}(r) \times c^{2}$ which gives a nuclear potential: $V(r)$, in an eulerian form, having a variation according to equation:

$$
V_{n}(r)=u_{i} P_{n}=V_{n}^{0} \cdot e^{-r / \eta^{*}} ; V_{n}^{0}=u_{i} P_{n}^{0}=\left(u_{i} / 2\right) \rho_{n}^{0} \times c^{2}
$$

With: $\eta^{*}=0.755 \mathrm{fm} ; v_{i}(0.6 \mathrm{fm}) \approx 0.9 \mathrm{fm}^{3}$ - the impenetrable quantum volume of the nucleon.

g. The neutron results in CGT by a specific "dynamid" model, with a degenerate electron with degenerate magnetic moment: $\mu_{e}{ }^{s}=-4.597 \mu_{N}$, rotated inside the quantum volume of a proton by the etherono-quantonic vortex $\Gamma_{P}$ of its magnetic moment, $\mu_{p}$ with a speed $v \approx 1.7 \times 10^{-2} c$, to an orbital with a radius: $r^{*} \approx 1.283 \mathrm{fm}$ under dynamic equilibrium of forces on tangent and radial directions. ${ }^{3,4}$

h. By CGT was argued the fact that- in a cold genesis model, the preon $z^{0}$ may results as cluster of $\mathrm{n}=42$ degenerate electrons with the mass: $m_{e}{ }^{*} \approx 0.8 m_{e}$ and with the super-dense kernels (centroids) vortexially confined in a volume of radius $r_{z}<0.2 \mathrm{fm}$, the $z^{0}$ cluster being given as a pair of quarcins: $c^{{ }^{*}}=21 \mathrm{~m}_{e}{ }^{*}$.

According to the model, considering also the existence of the zerons: $z_{p}=\left(z_{1}+z_{2}\right)=7 z^{0} ; z_{1}=3 z^{0}, z_{2}=4 z^{0}$, the deduced quasi-crystalline structure of the $z^{0}$ preon explains the value of the resulted masses of cold formed quarks, mesons and baryons (Table 1), by a quasi-crystalline model of quark (Figure 1) and by a baryon mass equation: ${ }^{5}$

$$
M_{b}=\mathrm{M}_{\mathrm{q}}+\mathrm{k} \cdot \mathrm{z}_{\pi}+\mathrm{n} \cdot(\mathrm{k}-6) \cdot \mathrm{z}_{2} ; \mathrm{M}_{\mathrm{q}}=\sum_{\mathrm{i}=1}^{3} \mathrm{~m}_{\mathrm{i}} ; \mathrm{m}_{\mathrm{i}}=\left(\mathrm{m}_{1}^{+} ; \mathrm{m}_{2}^{-}\right) ; \mathrm{k}=6 \div 9 ; \mathrm{n} \leq 2
$$

Table I Elementary particles: (theoretic mass) / (experimentally determined mass); $\bar{s}=s$ antiquark)

$$
\begin{array}{ll}
\text { Mesons: (q- q) } & \text { Baryons } \\
\begin{array}{ll}
\mu^{-}=2 Z_{1}+e^{-}=205 m_{e} / \mu^{+}=206.7 m_{e} & p_{r}=2 p+n=1836.2 m_{e} ; n_{e}=2 n+p=1838.8 m_{e} ; / p_{r}^{+}, n_{e}=1836.1 ; 1838.7 m_{e} ; \\
\pi^{o}=m_{1}+\bar{m}_{1}=270.4 m_{e} ; / \pi^{0}=264.2 m_{e} \pi^{+} & \Lambda^{o}=s+n+p=2212.8 m_{e} ; / \Lambda^{0}=2182.7 m_{e} \\
\pi^{+}=m_{1}+\bar{m}_{2}=273 m_{e} ; / \pi^{+}=273.2 m_{e} & \Sigma^{+}=v+2 p=2346.2 m_{e} ; \Sigma^{-}=v+2 n=2351.4 m_{e} ; / \Sigma^{+}, \Sigma^{-}=2327 ; 2342.6 m_{e} \\
\pi^{+}=m_{1}+\bar{m}_{2}=273 m_{e} ; / \pi^{+}=273.2 m_{e} & \Sigma^{o}=v+n+p=2348.8 m_{e} / \Sigma^{0}=2333 m_{e} ; \\
K^{o}=m_{2}+\bar{\lambda}=989.6 m_{e} ; / K^{o}=974.5 m_{e} & \Xi^{o}=2 s+p=2586.8 m_{e} ; \Xi^{-}=2 s+n=2589.4 m_{e} ; / \Xi^{0}, \Xi^{-}=2572 ; 2587.7 m_{e} ; \\
\eta^{o}=m_{2}+\bar{s}=1125.6 m_{e} ; / \eta^{0}=1073 m_{e} ; & \Omega^{-}=3 v=3371.4 m_{e} ; / \Omega^{-}=3278 m_{e} \cdot \\
\text { Basic quarks: } m_{1}=\left(z_{2}-m_{e}{ }^{*}\right)=135,2 m_{e}, & m_{2}^{-}=m_{1}+e^{-}+\sigma_{e}=137,8 m_{e} ; m_{2}^{-} \rightarrow m_{1}+e^{-}+\bar{v}_{e} ;\left(s_{e}=\left(e^{+*}+e^{-*}\right) \rightarrow \bar{v}_{e}\right) \\
\text { Derived quarks: } p^{+}\left(n^{-}\right)=m_{1}\left(m_{2}\right)+2 z_{p} & n^{-}=p^{+}+e^{-}+\sigma_{e} \rightarrow p^{+}+e^{-}+\bar{v}_{e} ; \lambda^{-}=n^{-}+z_{\pi^{;}} ; s^{-}=\lambda^{-}+z_{2} ; v^{-}=\lambda^{-}+2 z_{2}
\end{array}
\end{array}
$$

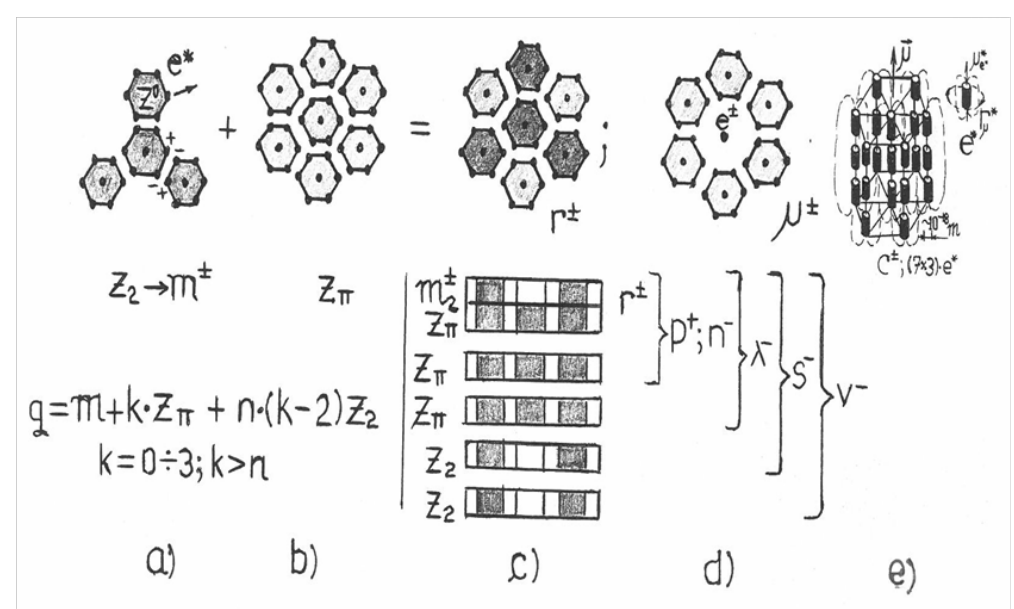

Figure I a), b)- quasi-crystallin form of $\mathrm{m}$-quark and $z_{\pi}$-zeron ; c) quasi-crystallin structure of cold formed quarks; d) quasi-crystallin ring form of $\mu^{ \pm}$; e) quasi-crystallin form of the quarcin $c^{ \pm}=21 m_{e}{ }^{*}$, with the kernel formed by superdense electronic centroids symmetrically disposed. 
i. The quarks confinement force is explained in CGT by a "bag" model given by the static quantum pressure gradient of the scalar charge of impenetrable quantum volume of the nucleon, ${ }^{6}$ which explains also its repulsive property observed in scattering interactions.

j. The theory also deduces a quasi-crystalline nuclear model that explains the Z, N "magical" nuclear numbers with maximum stability: $2 ; 8 ; 20 ; 28 ;(40) ; 50 ; 126$, as resulting from quasi-crystalline forms of alpha particles, with $Z=S\left(2 n^{2}\right)$, the ${ }_{82} \mathrm{~Pb}^{208}$ nucleus corresponding to the initial form: ${ }_{104} N^{208}\left(Z=2\left(4^{2}+6^{2}\right)\right)$ in which 22 protons was transformed into neutrons by $\beta^{-}$-emission giving $Z=82$, according to the model, which predicts a possible stable nuclear form with $A=4\left(5^{2}+7^{2}\right)=296$ nucleons. $^{4}$

k. Some exotic nuclear reaction such as the super-asymmetric nuclear reactions and some biological nuclear transmutations may be explained by the deuteron's self-resonance. ${ }^{3,4}$ The resulted vortexial atomic model is compatible with the hypothesis of the hydrino atom with $n^{-}=1 / 2$ sub-fundamental electronic level. ${ }^{?}$

The particle model of CGT may explain better the existent model of quasar' energy generation by matter-energy conversion, by the conclusion that the matter of the accretion disk, gradually pressed to the surface of a massive 'black hole' until a critical pressure: $p_{c}=n_{0} k_{B} T_{c}$ corresponding to a temperature $T_{c} \rightarrow T_{N} \approx 10^{13} \mathrm{~K}$ -of nucleons but also of quarks destruction, is transformed into radiation including also gamma rays and neutrins but generating also an emissions of quantons and of sinergonic etherons resulted from the energy $\varepsilon_{\gamma}=2 m_{e} c^{2}$ of destroyed gammonic pairs: $\gamma^{*}\left(e^{*_{+}}-e^{*_{-}}\right)$, generating in this way, periodically, an pulsatile anti-gravitic field and pseudo-charge which- according to the theory, explains the origin of the dark energy component which causes the Universe expansion. ${ }^{3,4}$

This natural theoretical conclusion is in concordance with a recent observational result of a team of astrophysicists from the University of Portsmouth that evidenced the existence of a dynamic character of the 'dark energy' at a level suggesting that the source of 'dark energy' is a dynamic field and not the quantum vacuum energy. ${ }^{8}$

The phenomenological model of cosmic expansion, resulted from CGT by the conclusion of pulsatile antigravitic cosmic fields existence, is with semi-sinusoidal variation of the expansion speed: $v(R)=v_{M} \times \sin \pi R / R_{u}$, as consequence of the intense matterenergy transformation processes especially in the zone of the expansion center and at the material Universe margins $R=R_{u}: 3,4$

$$
F_{e}=F_{a}-F_{d}=M_{s}^{*} d v_{e}=\frac{\pi \cdot v_{M}^{2} M_{s}^{*}}{2 R_{u}} \sin \frac{2 \pi R}{R_{u}} ; R<\frac{4}{5} R_{u} ; M_{s}^{*}=M_{s}^{0}\left(1-\frac{\mathrm{v}^{2}}{2 c^{2}}\right) ; \mathrm{v}_{M} \cong 0.5 c ; R \leq R_{u} / 6 \Rightarrow \mathrm{v}_{e} \approx \pi \frac{\mathrm{v}_{M} M}{R_{u}}=H \cdot R \quad \text { (4) }
$$

In CGT are explained microphysically also some magneto-electric and magneto-mechanic effects, such as: Einstein - De Haas, Barnett, Joffe-Kapitza, Aharonov-Böhm, Hooper-Monstein effects and the London's equations of superconductivity, by the hypothesis of the gravito-magnetic nature of the magnetic potential A, in particularby the conclusion that the modification of the canonic impulse of a moving electron: $\delta p= \pm e . A$ is given as a real impulse modifying by the dynamic sinergonic pressure of the $\Gamma_{A}$-vortex: $P=1 / 2 r c^{2}$, i.e by a (quasi)electric field: $E_{q}=\delta A / \delta t$ considered as being generated by the electron's entering in the field $\delta A= \pm A$ in a time $\delta$ t in which this field $E_{q} \sim \rho_{s} c^{2}$ acting over the electron, determines an impulse variation:

$$
\delta p_{e}=m_{e}\left(v_{f}-v_{i}\right)=m_{e} a . \delta t=e . E_{q} . \delta t=e . \delta A ; E_{q}=k_{1} \rho_{s} c^{2} \approx \delta A / \delta t ; \delta A \approx A(5)
$$

It results in consequence- according to $\mathrm{CGT},{ }^{3}$ that an identic effect is obtained also in the magnetic field of a star, but also for the electrogravitic charge of a neutral particle- phenomenon that may explain the possibility of the particles accelerating in the field of a pulsar or of a "black hole" type star- considered in the actual explicative model as a particularly gravitational effect generated near the event horizon. This hypothesis may be verified by an experiment of Aharonov-Böhm type made with photons, by passing a laser beam through a long optic fiber embedded on a coil housing in the center of which is placed a magnet or a strong electromagnet, measuring the frequency difference between the dextrogyre and levogyre sense of laser beam passing through the optic fiber.

\section{Conclusion}

The main conclusion resulted from the mentioned cold genesis theory (CGT) is that all basic structures, of electromagnetic radiation and of matter, can be generated 'at cold' by a vortexes cascade process initiated by chiral solitonic fluctuations in a cosmic fluid of protodark energy. According to CGT, the proto-dark energy is composed of quantons with $m_{h}=h / c^{2}=7.37 \times 10^{-51} \mathrm{~kg}$ giving the B-induction of magnetic field and etherons with $m_{g, s} \approx\left(10^{-70}, 10^{-60} \mathrm{~kg}\right)$ giving the gravitic and the gravito-magnetic field. By confining of quantonic superdense centroids in a very strong magnetic field were formed superdense centroids with bigger mass which generated stable vortexial structures of vectorial photons and of electrons with superdense centroid and photonic quantum volume. The magnetic confining of formed electrons and of negatron-positron pairs generated- at $T \rightarrow 0 K$, quasi-crystallin preons $z^{0}$ and cold formed quarks and elementary particles, formed as Bose-Einstein condensates, and thereafter- crystalline nuclear and atomic structures. The basic fields results also in CGT, from the particular kinetic of the proto-dark energy and of the vectorial photons, without the hypothesis of intermediary gluons- in case of the nuclear field, the quarks confining being explained by a specific "bag model", by the repulsive property of the surface of impenetrable quantum volume of the composed particle.

The phenomenological model of cosmic expansion results with semi-sinusoidal expansion speed variation, as consequence of the matter-energy conversion mainly in the expansion center and at the material Universe' margins, with the releasing also of the etheronic energy of the magnetic moments of degenerate electrons of component quarks, at temperatures of $\sim 10^{13} \mathrm{~K}$. The generated pulsatory antigravitic (pseudo)charge and field may explain the dynamic field nature of the dark energy component which generates cosmic expansion in concordance with some recent astrophysical observations, regarding also the gravitational wave detection.

The theory sustains also the conclusion that the local constancy of the elementary charge and magnetic moment values of elementary particles are ensured by the quantum vacuum negentropy, i.e. by quantum and sub-quantum winds of proto-dark energy, which explains also the functioning of some realized free energy devices.

\section{Acknowledgements}

My research project was partially or fully sponsored by (-none-) 
with grant number (-none-). In case of no financial assistance for the research work, provide the information regarding the sponsor.

\section{Conflicts of interest}

The author declares that there is no conflict of interest.

\section{References}

1. Krasznahorkay AJ, Csatlós M, Csige L, et al. Observation of Anomalous Internal Pair Creation in ${ }^{8}$ Be: A Possible Signature of a Light, Neutral Boson. Physical Review Letters. 2015;116(4):1-5.

2. Feng JL, Fornal B, Galon I, et al. Evidence for a Protophobic Fifth Force from ${ }^{8}$ Be Nuclear Transitions. Cornell University Library, USA. 2016.

3. Arghirescu M. He Cold Genesis of Matter and Fields. Education: Science Publishing Group, New York, USA. 2015.
4. Arghirescu M. A Quasi-Unitary Pre-Quantum Theory of Particles and Fields and Some Theoretical Implications. International Journal of High Energy Physics. 2015;2(4-1):80-103.

5. Arghirescu M. A preonic quasi-crystal quark model based on a cold genesis theory and on the experimentally evidenced neutral boson of 34 $\mathrm{m}_{\mathrm{e}}$. Global Journal of Physics. 2016;5(1):496-504.

6. Arghirescu M. A Correspondence with the Bag Model of a Pre-quantum B.-E. Condensate Model of Nucleon. International Journal of High Energy Physics. 2016;3(2):10-17.

7. Mills RL. Grand Unified Theory of Classical Physics. Vol. III, Millsian Inc, New Jersey, USA, 2011. 1566 p.

8. Zhao GB, Raveri M, Pogosian L, et al. Dynamical dark energy in light of the latest observations. Nature Astronomy. 2017;1(9):627-632. 